\title{
BIOINGENIERÍA DE TEJIDOS: CULTIVO DE QUERATINOCITOS HUMANOS EN EL TRATAMIENTO DE LA EPIDERMÓLISIS BULLOSA*
}

\author{
Drs. Nicolás Lorenzini V. ${ }^{1}$, Carlos Manterola D. ${ }^{1,2}$, Javier Moraga C. ${ }^{3}$, \\ Grupo MINCIR (Metodología e Investigación en CIRugía) \\ 1 Programa de Magíster en Ciencias Médicas, Mención Cirugía, Universidad de La Frontera. Temuco. \\ 2 Departamento de Cirugía y Traumatología, Universidad de La Frontera. Temuco. \\ 3 Universidad Autónoma de Chile. Santiago. \\ Chile.
}

\section{Abstract \\ Treatment of epidermolysis bullosa with allogeneic human cultured queratinocytes}

Background: Epidermolysis bullosa (EB) or "crystal skin" is a group of inherited disorders that affect the protein that forms the anchor between dermis and epidermis, producing blister injuries. Case report: We report a four years old boy with junctional EB and lesions in $80 \%$ of the body lasting 48 months. His right lower limb was treated with allogeneic human cultured queratinocytes during five weeks. After the treatment period, a re-epithelization of $90 \%$ of the intervened limb was observed. Its diameter increased from 23 to 27 $\mathrm{cm}$, the wound assessment scale score decreased from 30 to 13 and the visual analogue pain assessment score decreased from eight to two. Therefore allogeneic human cultured queratinocytes are a novel therapeutic alternative for EB.

Key words: Epidermolysis bullosa, Junctional $[\mathrm{MeSH}]$, Tissue engineering [MeSH], Cell culture Techniques [MeSH], Keratinocytes [MeSH], Wound and injuries [MeSH].

\section{Resumen}

Introducción: La epidermólisis Bullosa (EB) o "piel de cristal” es un grupo de trastornos hereditarios, el cual afecta las proteínas que forman la unión dermo-epidérmica de piel y mucosas, lo que lleva a la formación de lesiones ampollares. Objetivo: Comunicar la primera intervención con cultivos de queratinocitos humanos alogénicos (CQHA) en el tratamiento de la EB, en un centro de salud familiar de Chile el año 2013. Metodología: Se presenta el caso de un paciente de 4 años, quien presentaba lesiones en el $80 \%$ del cuerpo de 48 meses de evolución. Se realizó una intervención de la extremidad inferior derecha con el $90 \%$

\footnotetext{
Los autores no refieren conflictos de interés

Correspondencia: Dr. Nicolás Lorenzini V. nlorenziniv@gmail.com
}

*Recibido el 9 de septiembre de 2013 y aceptado para publicación el 22 de enero de 2014. 
comprometido con CQHA durante 5 sem con el fin de lograr la reepitelización y formación de piel indemne. Resultados: Posterior a las 5 sem del tratamiento se logró reepitelización del $90 \%$ de la extremidad intervenida, incremento del diámetro de la pierna de 23 a $27 \mathrm{~cm}$., en la escala de valoración de heridas se reduce de 30 a 13 puntos y en la escala de valoración análoga del dolor de 8 a 2 puntos. Conclusión: Se presenta una alternativa terapéutica para pacientes con EB.

Palabras clave: Epidermólisis Bullosa, bioingeneria de tejidos, cultivo alogénicos de queratinocitos, manejo avanzado de heridas.

\section{Introducción}

La epidermólisis bullosa (EB), corresponde a trastornos hereditarios (autosómicos o recesivos) de baja prevalencia. Su característica principal es la alteración de las proteínas que conforman la unión dermo-epidérmica; lo que produce una falla en la unión de epidermis y dermis, afectando a piel y mucosas. Esto, determina que los tejidos se separen ante traumas de baja intensidad con formación de ampollas, vesículas hemorrágicas y úlceras ${ }^{1}$.

Su prevalencia es de 32 casos por millón de habitantes, con una incidencia de 1,4 casos por millón por año ${ }^{2}$. En Chile la prevalencia es de 19,6 casos por millón de habitantes ${ }^{3}$; la fundación DebRA, que agrupa a estos pacientes, estimó que al año 2008 existían 156 pacientes con EB: 79 pacientes presentaban Epidermólisis Bullosa Simple (EBS); 8 Epidermólisis Bullosa de la Unión (EBJ); y 69 Epidermólisis Bullosa Distrófica (EBD). Durante los últimos años se ha agregado un nuevo subgrupo, el síndrome de Kindler, que se caracteriza por la aparición de lesiones mixtas ${ }^{4-5}$.

Si bien la clínica permite sospechar el diagnóstico, la biopsia por microscopía electrónica de transmisión (MET) confirma el subgrupo ${ }^{5-6}$.

Su tratamiento se centra en la prevención del estrés mecánico sobre la piel y mucosas para evitar la generación de nuevas lesiones. El tratamiento de las lesiones ya formadas busca la protección de la zona con apósitos activos para lograr una reepitelización por segunda intención.

Dependiendo del subtipo, presenta una elevada morbi-mortalidad por las complicaciones como deformaciones, amputaciones, neoplasias malignas, estenosis de la vía área e infecciones ${ }^{6}$. La EBS es la de mejor pronóstico a largo plazo, teniendo una mejoría clínica con los años. La EBD tiene una expectativa de vida de 20 a 40 años. Finalmente, la EBJ, que corresponde aproximadamente al $10 \%$ de los afectados, es la más letal y la expectativa de vida no supera los 3 años, con una mortalidad que va entre $40 \%$ a $87 \%$ dependiendo del subtipo ${ }^{7}$.

La técnica de cultivo de queratinocitos puede considerarse como un arma irreemplazable y una alternativa confiable para ser aplicada a pacientes con daño parcial o externo de su piel ${ }^{8}$.

El objetivo de este manuscrito, es comunicar la primera intervención con cultivos de queratinocitos humanos alogénicos (CQHA) en el tratamiento de la $E B$, en un centro de salud familiar de Chile el año 2013.

\section{Caso clínico}

Paciente de 4 años de sexo masculino portador de una EBJ, confirmada por estudio histológico y microscopia electrónica; con los siguientes diagnósticos asociados: estenosis de la vía área, desnutrición, anemia crónica, síndrome de inmovilización y dolor crónico con un componente mixto.

Antes del ingreso a curaciones en la atención primaria de salud, el paciente se encontraba en curaciones en su domicilio y en controles periódicos en la fundación DebRA, presentaba el $80 \%$ de su cuerpo cubierto con lesiones que se localizaban principalmente en tronco, pelvis y piernas; se movilizaba de manera de asistida.

El tratamiento de las lesiones consistió en someter al paciente a una ducha prolongada con el objeto de que la remoción de los apósitos produjera el menor estrés mecánico sobre la piel.

Los apósitos utilizados para realizar las curaciones fueron de espuma de poliuretano hidrófilo -que contiene un tensioactivo no iónico que es activado por la humedad, un humectante (glicerol) y un copolímero de almidón- que se utiliza para cubrir las lesiones de mayor extensión. En la zona pélvica y genital se utilizó una gasa parafinada estéril de baja adherencia, ya que la zona puede requerir varios cambios al día, además venda de gasas como apósito secundario.

Las lesiones de las piernas tenían cultivos positivos para Pseudomonas pyocyanea; y dado la escasa respuesta al tratamiento de antibióticos oral, se estaban realizando irrigaciones con una solución de ácido acético $0,05 \%$.

Dado la falta progresión en la reepilización de las lesiones, se realizó una revisión de la literatura y se planteó un cambio en el tratamiento avanzado de heridas. 


\section{Metodología}

Es la primera vez en Chile que se plantea una intervención para pacientes con esta patología con un apósito de queratinocitos humanos alogénicos crio-preservado. Debido al extenso compromiso que presentaba y el elevado costo del apósito, lo que hace imposible cubrir todo el cuerpo, se decidió intervenir la extremidad inferior derecha.

En las Figuras 1a y 1b se observa que el compromiso de la pierna derecha es del $80 \%$, con un puntaje en la escala de valoración de heridas de $30^{9}$, correspondiendo a lesiones enrojecidas de una profundidad menor a $1 \mathrm{~cm}$, exudativas, con exudado turbio, hipergranuladas asociado a tejido desvitalizados, dolor EVA 8, piel circundante macerada y sin signos de reepitelización.

Se iniciaron curaciones de la pierna derecha entre la zona infracondilea y el tobillo en un ambiente controlado, con un intervalo de 7 días entre las curaciones. Durante todo el período de tratamiento las curaciones consistieron en: baño de la zona y posterior aseo por arrastre con solución fisiológica para remover el tejido desvitalizado. En las dos primeras sesiones las lesiones se cubrieron con un apósito no tejido que contiene sulfato de plata en concentración de $8 \mathrm{mg} / \mathrm{g}$ de apósito, para disminuir la colonización por Pseudomonas pyocyanea.

Una vez confirmado un cultivo negativo para Pseudomonas pyocyanea, se inició el uso del apósito biológico con queratinocitos humanos, asociado a un apósito hidrocelular o espuma de poliuretano constituido por tres capas (una de film de poliuretano, una capa de espuma y una capa de contacto) no adhesivo; de manera de disminuir la fricción y adherencia y proteger la piel indemne. Finalmente, como apó- sito secundario se utilizó venda gasa, como fijación.

Las lesiones menores a $1 \mathrm{~cm}$ de diámetro mayor se protegieron con una malla de acetato de celulosa, impregnada con una emulsión de petrolato; tras lo cual, se observó una adecuada granulación (en caso contrario se utilizó una matriz hecha de $55 \%$ colágeno, $44 \%$ celulosa oxidada regenerada y $1 \%$ de plata oxidada regenerada lo cual acelera la formación de tejido de granulación y el apósito secundario puede ser venda gasa o gasa tejida, los que se fijaron con cinta con adhesivo de silicona que permite proteger la piel).

\section{Resultados}

El protocolo antes señalado, con apósitos con CQHA se mantuvo durante $5 \mathrm{sem}$. En las Figuras 2a y 2 b, se aprecia el avance logrado al finalizar la segunda semana de tratamiento.

Al aplicar la escala de valoración de heridas, el puntaje obtenido fue de 13 puntos. Se logró reepitelización, reducción de lesiones activas en la extremidad inferior derecha de un $90 \%$ a un $10 \%$ del total de la extremidad (el análisis de las imágenes se realizó utilizando el software Image J para Mac OS X Lion), exudado ausente y una reducción del dolor de un EVA 8 a un EVA de 2; recuperación de masa muscular con un incremento del diámetro de la pierna de 23 a $27 \mathrm{~cm}$.

Al final del período de tratamiento $(5 \mathrm{sem})$, se observó una clara disminución de las lesiones, con formación de piel indemne, sin embargo, se produjo una lesión del pie posterior al inicio del tratamiento, por lo que no se incluyó en el manejo realizado con CQHA (Figuras $3 a$ y $3 b$ ).

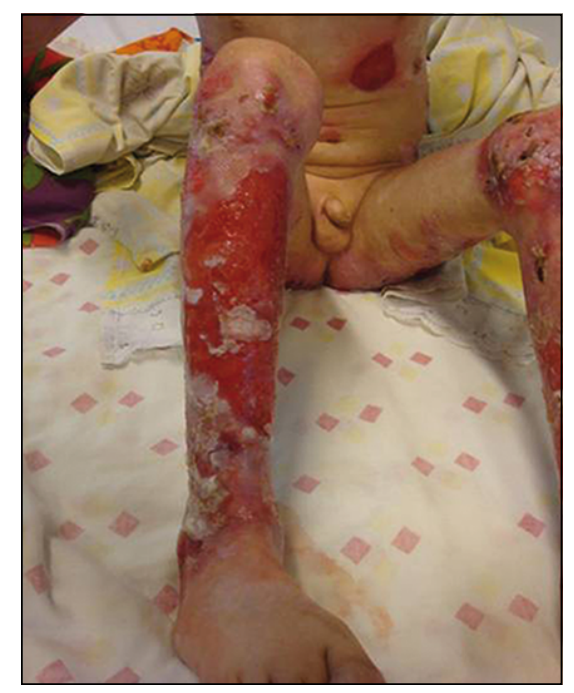

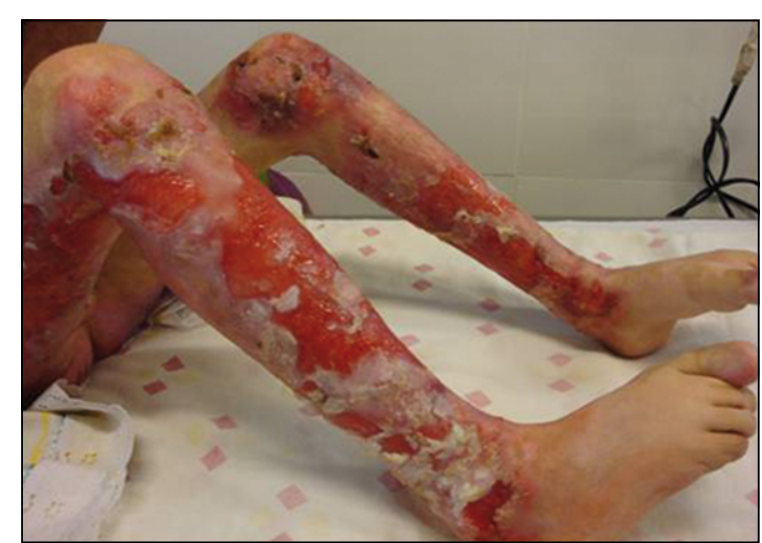

Figura 1a y 1b. Cara anterior y lateral de la pierna derecha al momento del diagnóstico. 

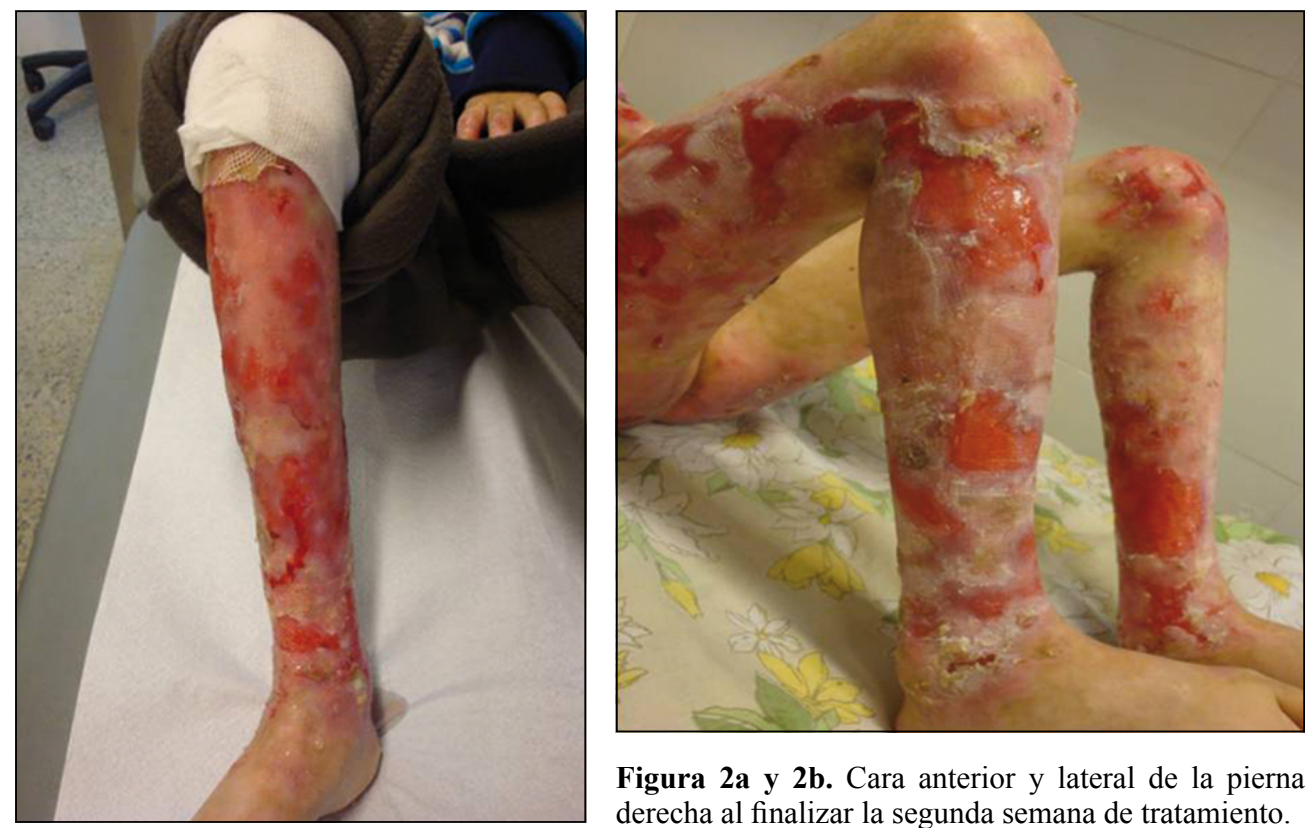

Figura 2a y 2b. Cara anterior y lateral de la pierna derecha al finalizar la segunda semana de tratamiento.
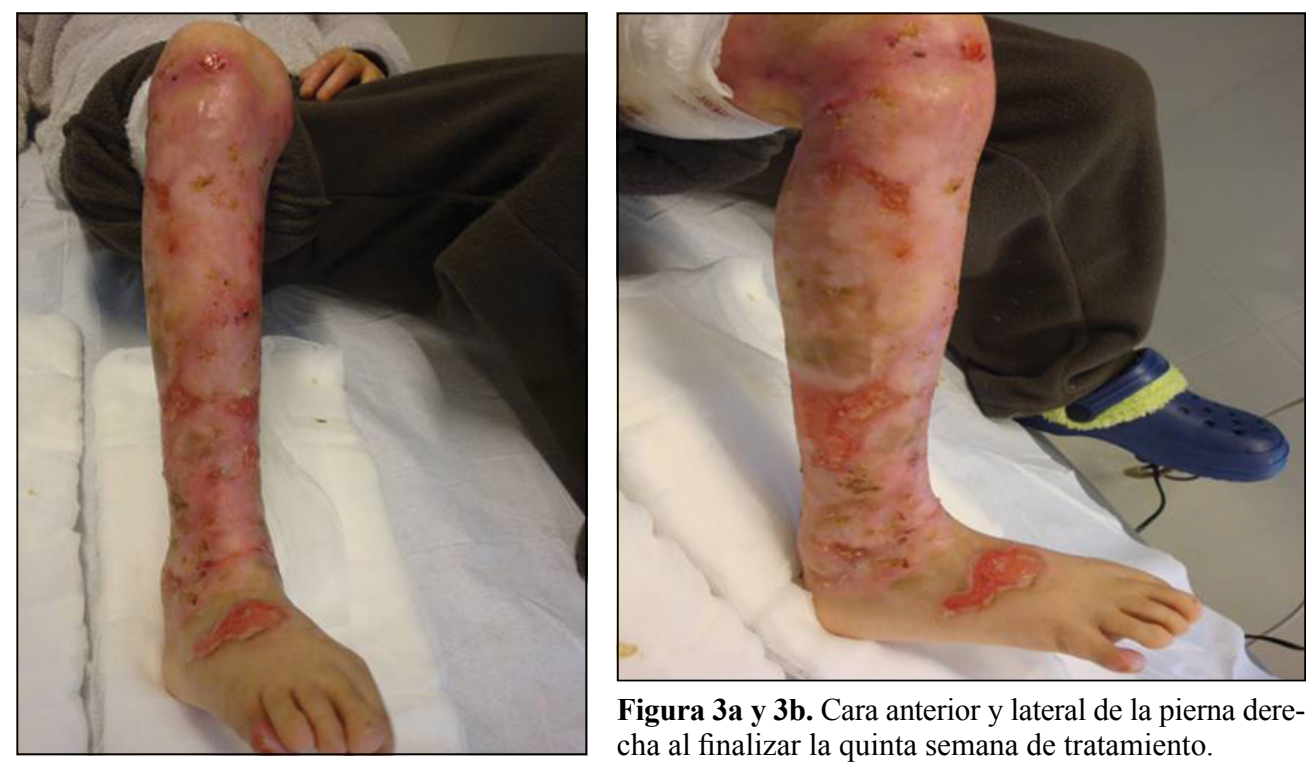

Figura 3a y 3b. Cara anterior y lateral de la pierna derecha al finalizar la quinta semana de tratamiento.

Posterior a la reepitelización de las lesiones se protegió la piel con espuma de poliuretano de manera de evitar recidivas.

La intervención terapéutica también consideró la rehabilitación kinesiológica, con lo cual se logró una deambulación autovalente; y derivación a terapia dental. Todo en la atención primaria de salud.

\section{Discusión}

Los CQHA en la actualidad han sido ampliamente utilizados en el manejo de pacientes quemados, úlceras venosas, pie diabético y úlceras por presión con muy buenos resultados ${ }^{10-12}$. De acuerdo a la revisión de la literatura, este tipo de terapia nunca 
había sido utilizado para el tratamiento de este tipo de patologías en Chile.

Los resultados han sido prometedores, considerando que en este caso en particular nunca se había logrado tener piel indemne en zona intervenida; esto producto de que las células alogénicas que no presentan la mutación ni generan respuesta inmune, permiten formar piel ya que las proteínas de anclaje de la unión dermoepidérmica son funcionales. Sin embargo, la vida media de estos queratinocitos es sólo de 28 días.

Los cultivos de queratinocitos son una alternativa para el manejo agudo de estas lesiones, de manera de lograr cubrir lesiones activas en cortos períodos de tiempo, para disminuir la morbilidad asociada. Es necesario tener presente que este no es un tratamiento curativo para esta patología, ya que no cambia la condición del paciente y en estas lesiones reepitelizadas posteriormente se deben tomar medidas preventivas para evitar la recidiva o la formación de nuevas lesiones.

Además como toda nueva biotecnología, los apósitos biológicos con CQHA tienen un costo económico elevado, por lo que su utilización debería ser considerada caso a caso.

Entre las opciones de terapia que en la actualidad buscan una curación definitiva están los estudios enfocados en terapia génica, utilizando terapias virales y no virales probadas en cultivos de tejidos, cuyo objetivo final es introducir genes que produzcan una modificación permanente en los queratinocitos. Otra arista en el tratamiento es la terapia celular, consistente en introducir una célula madre capaz de producir todas las proteínas en déficit inyectándola por vía sanguínea para que colonice toda la piel ${ }^{13}$.

Dado que la EB es una patología que no tiene cura; el tratamiento aplicado es una propuesta de manejo basada en la evidencia, la práctica clínica y los avances tecnológicos en el manejo avanzado de heridas y equipos especializados en la atención primaria de salud.

\section{Referencias}

1. DermIS [serial online] 1996-2007 [citado 13 Feb 2007];
1 (1): [2]. Disponible en URL: http://www.dermis.net/ dermisroot/es/40853/diagnose.htm.

2. McKenna KE, Walsh MY, Bingham EA. Epidermolysis bullosa in Northern Ireland. Br J Dermatol. 1992;127:318-21.

3. Rodríguez FA, Gana MJ, Yubero MJ, Zillmann G, Krämer SM, Catalán J, et al. Novel and recurrent COL7A1 mutations in Chilean patients with dystrophic epidermolysis bullosa. J Dermatol Sci. 2012;65:149-52.

4. Fine JD, Eady RAJ, Bauer EA, Bauer JW, BrucknerTuderman L, Heagerty A, et al. The classification of inherited epidermolysis bullosa (EB): Report of the Third International Consensus Meeting on Diagnosis and Classification of EB. J Am Acad Dermatol. 2008;58:931-50.

5. Yubero MJ, Krämer, DC, Schwartz R, Avello E. Manual Práctico: Cuidados básicos en pacientes con Epidermolisis Bullosa. $1^{\circ}$ Edición 2008, Fundación Debra Chile.

6. Horn HM, Tidman MJ. The clinical spectrum of epidermolysis bullosa simplex. Br J Dermatol. 2000;142:46872.

7. Epstein EH Jr. Molecular genetics of epidermolysis bullosa. Science 1992;256:799-804.

8. Bello G, Merentes E, Arvelo F. Cultivo de queratinocitos humanos in vitro. Gac Méd Caracas 1998 - anm.org. ve.

9. Aburto I, Leal C, Morgado P. Guía de Manejo y Tratamiento de Heridas. Valoración y Clasificacion. Ministerio de Salud de Chile. Año 2000.

10. O'Donnell TF Jr, Lau JA. Systematic review of randomized controlled trials of wound dressings for chronic venous ulcer. J Vasc Surg. 2006;44:1118-25.

11. Pham C, Greenwood J, Cleland H, Woodruff P, Maddern G. Bioengineered skin substitutes for the management of burns: a systematic review. Burns 2007;33:946-57.

12. Langer A, Rogowski W. Systematic review of economic evaluations of human cell-derived wound care products for the treatment of venous leg and diabetic foot ulcers. BMC Health Serv Res. 2009;9:115.

13. Pfendner EG, Bruckner A, Conget P, Mellerio J, Palisson F, Lucky AW. Basic science of epidermolysis bullosa and diagnostic and molecular characterization: Proceedings of the IInd International Symposium on Epidermolysis Bullosa, Santiago, Chile, 2005. Int J Dermatol. 2007;46:781-94. 